\title{
Reentrant Phase Transition in Charged Colloidal Suspensions
}

\author{
Akhilesh K. Arora, B. V. R. Tata, A. K. Sood, and R. Kesavamoorthy \\ Materials Science Laboratory, Indira Gandhi Centrefor Atomic Research, Kalpakkam 603102, India
}

\begin{abstract}
We report the observation of a novel phase transition in dilute aqueous suspensions of polystyrene particles as a function of ionic impurity concentration $C$. The suspension phase separates into dense and rare phases only for a restricted range of $C$ which depends on particle concentration $n$. The dense phase has liquidlike or crystalline order depending on $n$ and $C$. Free energies of the homogeneous and the phase-separated states are calculated with an effective interparticle potential. The calculated phase diagram is in qualitative agreement with the present experimental results.
\end{abstract}

Electrostatically stabilized aqueous colloidal suspensions can develop structural orders similar to those in atomic liquids, ${ }^{3}$ crystalline solids, ${ }^{2}$ and even glasses ${ }^{3,4}$ when suitably deionized and hence are model systems to study cooperative behavior in condensed matter. It is widely believed that the particles interact predominantly via a screened Coulomb potential ${ }^{5} U(r) \sim \exp (-k r) / r$ where $r$ is the interparticle separation and $k$ is the inverse Debye screening length given by $\kappa^{2}=4$ re ' ( $\boldsymbol{Z} \boldsymbol{Z}$ $+\boldsymbol{C} z$ ?) $\boldsymbol{l} \boldsymbol{c} \boldsymbol{k} \sim \boldsymbol{T}$. The first term arises from $\boldsymbol{n} \boldsymbol{Z}$ counterions coming from the particles with concentration $\mathrm{n}$ and the second term is the contribution of impurity ions with concentration $C$ and charge ezi. $c$ is the dielectric constant of the medium and $T$ the temperature. The feature that is unique to these systems is that one can alter the strength and the range of the interaction simply by changing $C$ or $n$. There also exists a primary minimum6 in the interparticle interaction due to London-van der Waals attraction. This minimum is important when the Coulomb barrier, which decreases as $C$ increases because of screening, is not strong enough to prevent the particles from coming in contact with each other, giving rise to irreversible aggregation (flocculation). However, when $C$ is low such that the Coulomb barrier does not allow the particles to come close to each other, this attraction is not important and the suspension is said to be electrostatically stabilized.

In this Letter we report a novel phase transition in monodisperse aqueous suspensions of polystyrene particles as a function of impurity concentration. The suspension phase separates into a dense (concentrated) and a rare (dilute) phase only for a restricted range of $C$ and remains homogeneous on either side of this range. A system consisting of particles interacting via a purely repulsive potential when restricted to a finite volume is expected to remain homogeneous except for the fluid. crystal transition. Phase separation into dense and rare phases would necessarily imply a secondary minimum in the interaction potential. In a recent theory of electrostatic interaction in colloidal suspensions ${ }^{7}$ an effective interaction potential $W(r)$ is obtained which takes the counterion-polyion (particle) interaction also into account. $W(r)$, apart from the screened Coulomb repulsion, has an attractive term leading to a secondary minimum whose position and depth are controlled by $\mathrm{C}$. In order to understand the phenomena theoretically, we calculate the free energies of the homogeneous and the phase-separated states using the effective pair potential $W(r)$. The two phase boundaries predicted by the calculations (the reentrant behavior) are physically explained. It may be mentioned that reentrant phase separations have been observed in binary liquids ${ }^{8}$ and other multicomponent systems like microemulsions ${ }^{9}$ where temperature is the control parameter.

Monodisperse suspensions of polystyrene particles of 0.109 and $0.16 \mu \mathrm{m}$ diam $^{10}$ were prepared in deionized water by dilution of the stock suspensions to the desired concentration. The suspensions thus prepared were in the homogeneous state and had conductivity of the order of a few tens of microsiemens per centimeter. Suspensions were in cylindrical glass cells which were placed in an index-matching bath of glycerene. In order to measure the transmittance of the suspension a $2 \cdot \mathrm{mW} \mathrm{He}-\mathrm{Ne}$ laser was focused into the cell along its diameter and the transmitted intensity was measured with an EG\&G photodiode (model SGD-100A). The cell was moved vertically with a translation stage to measure the height dependence of transmitted intensity. The structure factor of the suspension was measured at various heights in the cell by measurement of angle-resolved polarized-light scattering with a setup described earlier."

In order to reduce the impurity concentration a mixed bed of ion-exchange resins (cation, Dowex 50W-X4; anion, Dowex 1-X4) was added to the suspension. The resins settle down at the bottom of the cell. As $C$ reduces because of the action of the ion-exchange resins, the homogeneous suspension with particle concentration $n$ undergoes phase separation. Phase separation was found to occur even in the suspensions which were shaken occasionally to remove the gradient in $C$. The dense phase with particle concentration $n_{d}$ much higher than $\mathrm{n}$ settles below because of gravity (density of the polysty- 
rene particles, $1.05 \mathrm{~g} \mathrm{~cm}^{-3}$, being higher than that of water), leaving the rest of the volume occupied by the rare phase with particle concentration $n_{r}\left(n_{r}<n\right)$. Figure 1(a) shows the height dependence of the transmitted intensity in a suspension with $n=4.2 \times 10^{12} \mathrm{~cm}^{-3}$ at various times after addition of the ion-exchange resins. The horizontal sections of the curves $a-d$ represent the rare phase and the sedimented dense phase is identified by the sudden decrease of the transmitted intensity. A part of the dense phase showed Bragg diffraction of the laser beam, on indication of the presence of crystalline order. Angle-resolved polarized-light scattering from the part of the dense phase which did not show iridescence and from the rare phase showed that the former had strong liquidlike order whereas the latter did not have any structural order. The coexistence of crystalline and liquid orders in the dense phase was due to a gradient in $n$ within the dense phase arising from gravitational effects. ${ }^{12}$ The attenuation of the laser beam through the dense phase does not faithfully represent the particle concentration profile because of multiple scattering; however, in the rare phase it can be used to estimate $n_{r}$. The attenuation in the rare phase decreases as the grains (domains) of the dense phase settle down under gravity. Figure 1(b) shows the time evolution of $n_{r}$. Note from Fig. 1(b) that the settling of the dense-phase grains is nearly complete in less than ten hours; however, the volume occupied by the dense phase continues to increase (interface between the dense and the rare phases continues to move upwards) even afterwards as impurities are further reduced by the action of the resins. Figure 1(c) shows the position of the interface as a function of time. In approximately a hundred hours the dense phase occupies the entire volume and the system again becomes a single phase except for the gradient in $\mathrm{n}$ due

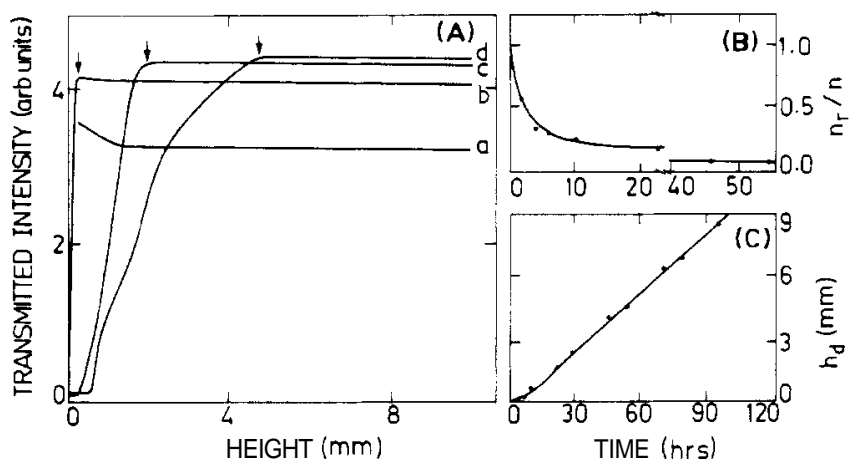

FIG. 1. (a) Transmitted intensity as a function of height in the suspension cell containing 0.109 -pm-diam particles at $n=4.2 \times 10^{12} \mathrm{~cm}^{-3}$ at different times after the addition of the ion-exchange resins: $\boldsymbol{a}, 0.1 \mathrm{~h} ; \boldsymbol{b}, \mathbf{4 . 2} \mathrm{h} ; \mathrm{c}, \mathbf{2 2} \mathrm{h} ; \boldsymbol{d}, \mathbf{5 4} \mathrm{h}$. Vertical arrows indicate the position of the interface between the dense and the rare phases. (b) Concentration in the rare phase relative to $n$ as a function of time. (c) Height of the dense phase $\left(h_{d}\right)$ as a function of time. to gravitational effects. This suggests that the average interparticle separation in the dense phase continues to change (increase) as $C$ decreases. This is confirmed from the observed decrease in the Bragg angles from the crystalline phase and the position of first peak in the liquid-structure factor from the dense phase. It needs to be emphasized that the particles in the reentrant homogeneous state obtained after deionization interact strongly, whereas those in the initial homogeneous state are essentially noninteracting. Similar behavior is seen in suspensions with other particle concentrations and also in those of other diameter particles.

In order to understand the reentrant behavior of the phase transition, we calculate the free energies of the homogeneous and the phase-separated states. Consider first a homogeneous suspension of $N$ particles each of diameter $2 a$ in a volume $V(n=N / V)$ interacting via the effective interparticle potential $W(r)$. The average interparticle separation $\boldsymbol{R}$ can be related to $\boldsymbol{n}$ as $\boldsymbol{R}$ $=(\pi n / 6)^{-1 / 3}$. The free energy $F_{h}$ of the homogeneous state can then be written as

$$
F_{h}=\frac{1}{2} p N W(R)-\mathrm{Tk}_{\mathrm{B}} N s(f),
$$

where $\boldsymbol{p}$ is the number of nearest neighbors and the effective potential $W(r)$ is given as ${ }^{?}$

$$
W(r)=\left(\frac{e Z \sinh (k a)}{k a}\right)^{2}\left(\frac{A}{r}-\frac{k}{2}\right) \frac{\exp (-k r)}{\epsilon},
$$

where $A=1+k a \operatorname{coth}(k a)$. The first and second terms are repulsive and the attractive parts of the potential. The repulsive part dominates at small distances. This effective potential has been obtained by our taking the particle-particle repulsion, the small-ion-particle attraction, and the small-ion-small-ion repulsion into account and integrating out all the degrees of freedom of small ions.' The second term in Eq. (1) is the contribution of entropy and is obtained in a straightforward manner ${ }^{13}$ from the number of possible ways in which $N$ particles can be distributed in $N_{a}$ cells each of an equivalent hard-sphere volume $v_{\text {hs }}\left(N_{a}=V / v_{\text {hs }}\right)$. The effective volume fraction is then $f=n v_{\text {hs }}$ and the function $s(f)$ is given as ${ }^{13}$

$$
s(f)=f^{-1} \ln \left(f^{-1}\right)-\left(f^{-1}-1\right) \ln \left(f^{-1}-1\right) .
$$

As the Coulomb barrier [the first term in Eq. (2)] does not allow the particles to come close to each other, it is meaningful to visualize the particles as hard spheres of equivalent diameter $r_{\mathrm{hs}}\left(v_{\mathrm{hs}}=\pi r_{\mathrm{hs}}^{3} / 6\right)$ for the purpose of the calculation of the entropy. The hard-sphere diameter is obtained as ${ }^{6}$

$$
r_{\mathrm{hs}}=2 a+\int_{2 a}^{r_{0} 0}\left\{1-\exp \left[-W(r) / k_{\mathrm{B}} T\right]\right\} d r,
$$

where $r_{0}$ is the distance at which $W(r)$ goes to zero.

As mentioned earlier the potential $W(r)$ has a minimum at $r_{m}$ and consequently it is possible, in princi- 
ple, for us to lower the interaction energy by bringing the particles into the potential well. Note that this can happen only when $R>r_{m}$, which is given as'

$$
r_{m}=\left\{A+[A(A+2)]^{1 / 2}\right\} / k \text {. }
$$

In the phase-separated state the particles in the dense phase are assumed to be staying in the potential minimum. The particle concentration in the dense phase is then $n_{d}=6 / \pi r_{m}^{3}$. If $x N$ particles out of the total $\mathrm{N}$ are present in the dense phase, the volume occupied by them is $x N / n_{d}$ and the remaining $(1-x) N$ particles are assumed to be uniformly distributed in the rest of the volume to constitute the rare phase. The particle concentration in the rare phase is then $n_{r}=(1-x)[1 / n$ $\left.-x / n_{d}\right]^{-1}$ and the average interparticle separation $r_{2}$ in the rare phase becomes $\left(\pi n_{r} / 6\right)^{-1 / 3}$. The free energy of the phase-separated states, $F_{\text {ps }}(x)$, which depends on the fraction $\mathrm{x}$, can now be written as

$$
F_{\mathrm{ps}}(x)=E_{\mathrm{ps}}(x)-T S_{\mathrm{ps}}(x),
$$

where the interaction energy is

$$
E_{\mathrm{ps}}(x)=\frac{1}{2} N\left[x p_{1} W\left(r_{m}\right)+(1-x) p_{2} W\left(r_{2}\right)\right],
$$

where $p_{1}$ and $p_{2}$ are the numbers of nearest neighbors in the dense and rare phases, respectively. The entropy is

$$
S_{\mathrm{ps}}(x)=k_{\mathrm{B}} N\left[x s\left(f_{1}\right)+(1-x)_{s}\left(f_{2}\right)\right],
$$

where $f_{1}=n_{d} v_{\text {hs }}$ and $f_{2}=n_{r} v_{\text {hs }}$ are the effective volume fractions in the dense and rare phases, respectively. The phase separation will occur if $\Delta F(x)=F_{\mathrm{ps}}(x)-F_{h}<0$.
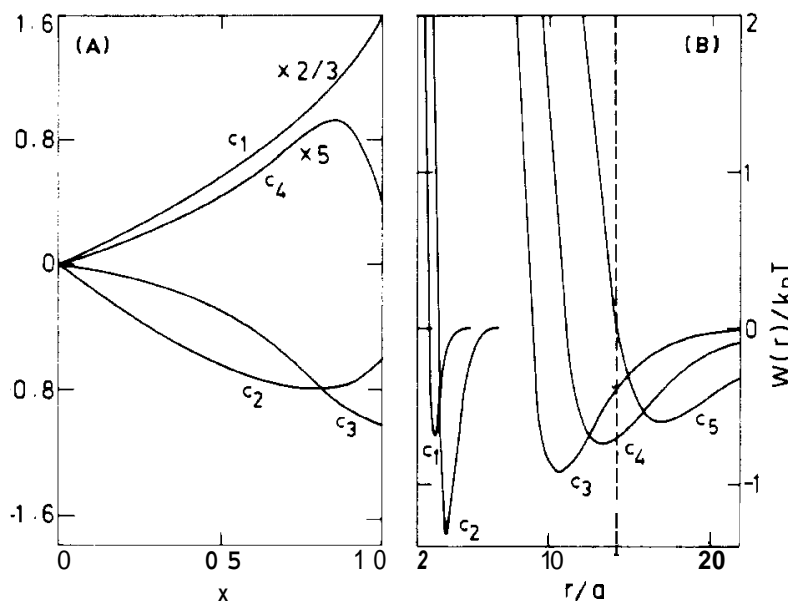

FIG. 2. (a) The difference in the free energy $\Delta F(x)$ relative to the thermal energy $N k_{\mathrm{B}} T$ as a function of the fraction $x$ for various impurity concentrations $C$ expressed relative to the counterion concentration $n Z(c=C / n Z): c_{1}=170, c_{2}=60$, $c_{3}=3$, and $c_{4}=1.5$. The value of $\phi$ is $0.284 \%$. (b) The effective interparticle potential $W(\boldsymbol{r})$ relative to $k_{\mathrm{B}} T$ for various values of $c_{.} c_{1}$ to $c_{4}$ : same as those in $(a) ; c_{5}=0.5$. Vertical dashed line shows the position of average interparticle separation $\mathrm{R}$ for $\phi=0.284 \%$.
Figure 2(a) shows $\Delta F(x)$ in units of the thermal energy $N k_{\mathrm{B}} T$ as a function of $\mathrm{x}$ for various impurity concentrations for $n=4.2 \times 10^{12} \mathrm{~cm}^{-3}$ which corresponds to an actual volume fraction $\phi=0.284 \%\left(\phi=4 \pi a^{3} n / 3\right)$. The particle (polyion) valency $\boldsymbol{Z}$ is taken as 500 which is the same as the reported value for particles of this diameter. ${ }^{4}$ The numbers of nearest neighbors, which could be different for different phases, enter into the calculations as parameters. In order to keep the calculations free from the arbitrariness of parameters to the extent possible we take $p=p_{1}=p_{2}=8$ which corresponds to a bcctype coordination. This is reasonable as dilute suspensions are known to crystallize into bcc structures.* One can see from Fig. 2(a) that as C decreases $\Delta F(x)$ becomes negative and again becomes positive for smaller values of C. Figure 2(b) shows the potential $W(r)$ for different values of $\mathrm{C}$. Note that the depth and the position of the well depend strongly on $C$.

The phase diagram ( $\phi$ vs $C$ ) obtained from the condition $\Delta F(x)<0$ is shown in Fig. 3 and can be physically understood as follows. The suspension is homogeneous on the high-impurity side because the depth of the well is not large enough to trap the particles [see curve $c_{1}$ in Fig. 2(b)] and entropy forces the system to remain homogeneous. As one moves across the phase boundary $C_{h}$ the system undergoes phase separation and the particle concentration in the dense phase is dictated by the position of the potential well [see curves $c_{2}$ and $c_{3}$ in Fig. 2(b)]. The dashed curve in Fig. 3 corresponds to $r_{m}=R$. For values of $\mathrm{C}$ lower than that given by the dashed curve the average particle separation $\mathrm{R}$ lies on the repulsive edge of the potential and the system is expected to be homogeneous again [see curve $c_{s}$ in Fig. 2(b)]. The presence of the entropy term causes the phase boundary $C_{1}$ to be shifted from the $r_{m}=R$ curve making the impurity concentration $c_{4}$ (Fig. 3) lie in the region of

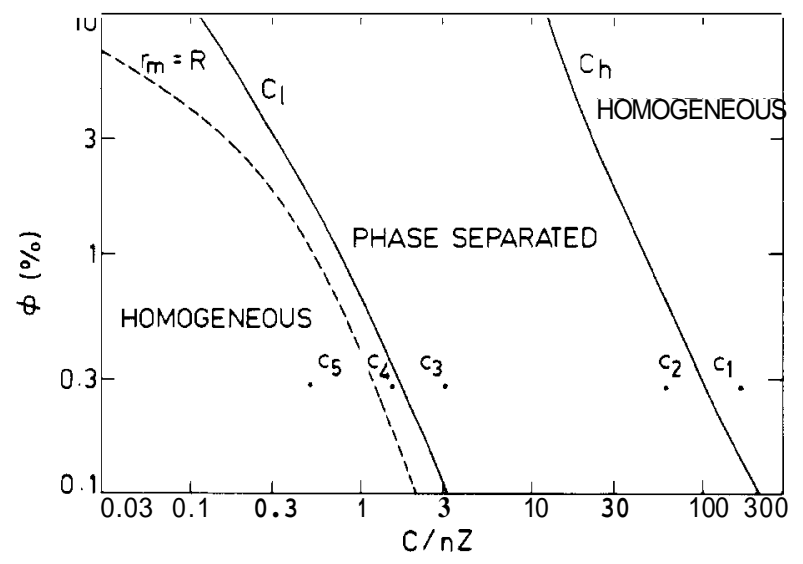

FIG. 3. Calculated phase diagram as a function of volume fraction $\phi$. The dashed curve corresponds to $r_{m}=R$. The points along $\phi=0.284 \%$ correspond to the impurity concentrations $c_{1}$ to $c_{5}$ of Fig. 2. 
homogeneous phase. The phase transition across the boundary $C_{h}$ is analogous to the condensation of a monatomic gas where one observes the dense (liquid) and the rare (vapor) phases coexisting; however, the phase boundary $C_{1}$ is entirely due to the strong dependence of $r_{m}$ on $\mathrm{C}$.

In a recent optical-microscopy investigation ${ }^{14}$ on colloidal suspensions of polystyrene particles of larger diameter, "ordered" (crystalline) and "disordered" (not having crystalline order) regions were found to coexist and the interparticle separation $r_{c}$ in the ordered region was found to be smaller than average interparticle separation $\boldsymbol{R}$ expected for homogeneous dispersion. This clearly suggests the presence of a dense phase similar to the present observations. The results were interpreted by our assuming that the crystalline order had formed because of the trapping of the particles in the potential well of $W(r)$, whereas the presence of disordered regions could not be understood.' The present experimental observations clearly demonstrate that the dense phase is not necessarily crystalline as assumed previously. ${ }^{7,14}$ Whether the dense phase has crystalline or liquidlike order or both is governed by the crystal-liquid phase diagram." In another similar study ${ }^{16}$ an increase of $r_{c}$ towards $\boldsymbol{R}$ is reported during the deionization process, which is in agreement with the present observations in the dense phase. The reentrant homogeneous state could not be observed in these investigations ${ }^{14.16}$ perhaps because of the large value of $\boldsymbol{Z}$.

In conclusion, a novel phase transition has been observed in aqueous suspensions of polystyrene particles as a function of impurity concentration. The free-energy calculations in the two states are able to explain the two phase boundaries. A secondary minimum in the effective interparticle potential leads to the phase transition.

${ }^{1}$ P. N. Pusey, Philos. Trans. Roy. Soc. London A 293, 429 (1979).

${ }^{2}$ R. Williams and R. S. Crandall, Phys. Lett. 48A, 225 (1974)

${ }^{3}$ P. N. Pusey and W. van Megen, Nature (London) 320, 340 (1986).

${ }^{4}$ H. M. Lindsay and P. M. Chaikin, J. Chem. Phys. 76, 3774 (1982)

${ }^{5}$ P. Pieranski, Contemp. Phys. 24, 25 (1983).

${ }^{6}$ C. A. Castillo, R. Rajagopalan, and C. S. Hirtzel, Rev. Chem. Eng. 2,237 (1984).

${ }^{7}$ I. Sogami and N. Ise, J. Chem. Phys. 81, 6320 (1984).

${ }^{8}$ A. W. Fransis, Liquid-Liquid Equilibrium (Interscience, New York, 1963).

${ }^{9}$ C. M. Sorensen, Chem. Phys. Lett. 117, 606 (1985).

${ }^{10}$ Obtained from Serva Feinbiochemica GmbH, Federal Republic of Germany.

IIA. K. Arora, J. Phys. E 17, 1119 (1984).

${ }^{12}$ R. Kesavamoorthy and A. K. Arora, J. Phys. A 18, 3389 (1985).

${ }^{13}$ R. Williams, R. S. Crandall, and P. J. Wojtowicz, Phys. Rev. Lett. 37, 348 (1976).

${ }^{14}$ K. Ito, H. Nakamura, and N. Ise, J. Chem. Phys. 85, 6143 (1986).

${ }^{15}$ D. Hone, J. Phys. (Paris), Colloq. 46, C3-21 (1985).

${ }^{16}$ T. Okubo, J. Chem. Phys. 86, 5182 (1987). 